\title{
Inhibitor of DNA binding 2 is a novel therapeutic target for stemness of head and neck squamous cell carcinoma
}

\author{
Woo Jin Bae ${ }^{1,7}$, Bon Seok Koo ${ }^{2,7}$, Sang Hyuk Lee ${ }^{3,7}$, Jin Man Kim ${ }^{4}$ Young Soo Rho ${ }^{5}$, Jae Yol Lim ${ }^{6}$, \\ Jung Hwa Moon ${ }^{1}$, Jae Hoon Cho ${ }^{1}$ and Young Chang Lim*,1 \\ ${ }^{1}$ Department of Otorhinolaryngology - Head and Neck Surgery, Research Institute of Medical Science, Konkuk University \\ School of Medicine, No. 1, Hwayang-dong, Gwangin-gu, 120-1 Neungdong ro, Seoul 143-752, South Korea; ${ }^{2}$ Department of \\ Otolaryngology - Head and Neck Surgery, Cancer Research Institute, Research Institute for Medical Sciences and Pathology, \\ Daejeon 301-721, South Korea; ${ }^{3}$ Department of Otorhinolaryngology - Head and Neck Surgery, Sungkyunkwan University School \\ of Medicine, Kangbuk Samsung Hospital, Seoul 110-746, South Korea; ${ }^{4}$ Chungnam National University College of Medicine, \\ Daejeon 301-721, South Korea; ${ }^{5}$ Department of Otolaryngology - Head and Neck Surgery, Hallym University College of Medicine, \\ Seoul 134-701, South Korea and ${ }^{6}$ Department of Otorhinolaryngology, Yonsei University School of Medicine, Seoul 06273, South \\ Korea
}

Background: Head and neck squamous cell carcinomas (HNSCCs) are highly lethal epithelial tumours containing self-renewal cancer stem cells (CSCs). CSCs in HNSCCs are strongly associated with tumour initiation, invasion, and chemoradiation resistance. However, the important factors regulating stemness in HNSCCs remain unclear. Here, we investigated the molecular roles and clinical significance of inhibitor of DNA binding 2 (Id2) protein to determine if it constitutes a novel therapeutic target for ablating HNSCC cells with stemness.

Methods: We performed in vitro and in vivo studies of Id2 function and its effects on stemness using HNSCC cells. We also examined whether Id2 expression could be used as a prognostic indicator through immunohistochemical staining of 119 human HNSCC tumours.

Results: Expression of Id2 was higher in HNSCC cells with stemness compared with differentiated HNSCC cells. Overexpression of Id2 increased proliferation, self-renewal, and expression of the putative stemness marker CD44 in HNSCC cells in vitro and in vivo. In contrast, silencing of Id2 using short hairpin RNA attenuated the stemness phenotype of HNSCC cells by reducing selfrenewal, CD44 expression, cisplatin chemoresistance, and xenograft tumourigenicity. Most importantly, increased expression of Id2 was closely associated with poorer post-treatment survival rates in HNSCC patients.

Conclusions: Inhibitor of DNA binding2 represents a novel and promising therapeutic target for treating and improving the clinical outcomes for patients with HNSCC.

Head and neck squamous cell carcinomas (HNSCCs) are one of several types of cancers originating from the upper aerodigestive tract, which includes the oral cavity, pharynx, and larynx. HNSCCs account for more than 300000 new cases worldwide annually (Leemans et al, 2011). Major risk factors associated with the development of HNSCCs are smoking, alcohol consumption, and

\footnotetext{
*Correspondence: Professor YC Lim; E-mail: ycliment@kuh.ac.kr

${ }^{7}$ These authors contributed equally to this work.
}

Received 3 July 2017; revised 17 September 2017; accepted 22 September 2017; published online 2 November 2017

(C) 2017 Cancer Research UK. All rights reserved 0007-0920/17 
human papilloma virus infection (Polanska et al, 2014). Although recent advances in diagnostic and therapeutic techniques for these tumours have been developed, survival has not markedly improved in the past decades because patients still frequently develop locoregional tumour recurrences, distant metastases, and secondary primary tumours (Guo and Califano, 2015). Thus, an improvement in the prognosis of patients with HNSCCs will require a deeper understanding of the molecular mechanisms underlying various steps in HNSCC tumourigenesis. These efforts may even result in the development of novel therapeutic strategies and a possible cure for HNSCCs.

Recent studies of the pathogenesis of HNSCCs have identified a sub-population of cancer cells, termed cancer stem-like cells (CSCs) or cancer-initiating cells. These cells are highly tumourigenic and resistant to conventional chemotherapy agents. They also display features commonly associated with embryonic or adult stem cells, such as self-renewal and multipotency (Matsui, 2016). Notably, in a clinical setting, CSCs appear to have a critical role in treatment resistance, resulting in tumour recurrence and metastasis. These are common causes of high mortality in patients with HNSCCs (Bhaijee et al, 2012). Thus, selective elimination of these CSCs constitutes one promising approach to treating HNSCCs.

Inhibitor of DNA binding (Id) proteins are basic helix-loophelix (bHLH) transcription factors. They constitute four subclasses, which include Id1, Id2, Id3, and Id4 (Lasorella et al, 2014). Other bHLH families regulate the transcription of various target genes, and function as either homodimers or heterodimers (Perk et al, 2005). However, because of the destitute DNA-binding domain, Id proteins bind to ubiquitously expressed $\mathrm{E}$ protein transcription factors or tissue-restricted bHLH proteins, preventing them from forming DNA-bound, transcriptionally active complexes (Sikder et al, 2003). As a result, Id proteins control multiple processes during normal development and adult life, including cell fate determination, differentiation, and proliferation (Desprez et al, 2003). Embryonic neural stem cells completely lose their selfrenewal capacity and multipotency in the absence of Id1, Id2, and Id3 (Jung et al, 2010; Niola et al, 2012). Inactivation of Id1 and Id3 triggers premature differentiation of neuronal stem cells in the developing mouse brain (Riechmann and Sablitzky, 1995; Jung et al, 2010).

Inhibitor of DNA binding proteins are often overexpressed in many human cancers. In addition, recent genomic analyses of cancer tissues identified various loss-of-function mutations in Id genes, suggesting that this family may have roles as tumour suppressors (Love et al, 2012; Richter et al, 2012). Numerous oncoproteins, such as MYC, RAS, epidermal growth factor (EGF), and basic fibroblast growth factor (bFGF), increase the transcriptional activity of Id proteins as upstream regulators (Lasorella et al, 2014). Furthermore, Id proteins are targeted for degradation by APC/C-CDH1 and SMURF2, which are two ubiquitin ligases that function as tumour suppressors (Wang et al, 2008; Kong et al, 2011). In addition, the expression of Id proteins can inform the prognosis for many human cancers (Lasorella et al, 2014).

Inhibitor of DNA binding proteins may be involved in tumourigenesis and maintenance of normal stem cell functions. Recent reports have established that Id proteins promote the formation of CSCs (Anido et al, 2010; O'Brien et al, 2012). In colon CSCs, the combined expression levels of Id1 and Id 3 increased during self-renewal and tumour initiation (O'Brien et al, 2012). Additionally, conditional deletion of Id1, Id2, and Id 3 in tumour cells decreased glioma stem cell populations (Anido et al, 2010). However, it is unclear whether Id proteins regulate CSCs in HNSCC. Here, we report that Id2 is essential for the maintenance of HNSCC CSCs and constitutes a potential therapeutic target for selectively eliminating HNSCC CSCs.

\section{MATERIALS AND METHODS}

Cell lines and reagents. We used three human HNSCC cell lines for this study. Of these, two HNSCC cell lines (FaDu and SNU1041) were obtained from the Korean Cell Line Bank and cultured in DMEM containing 10\% FBS and 1\% penicillin/ streptomycin. The third cell line (KU-SCC-3) was obtained from a primary culture of human HNSCC tumours. Briefly, the KU-SCC3 cells were isolated from patients' primary HNSCC tumours and placed into a serum-free conditional medium containing Dulbecco's modified Eagle's medium and Ham's F12 medium (DMEM/ F12) supplemented with human recombinant bFGF (10 $\mathrm{ng} \mathrm{ml}^{-1}$; R\&D Systems, Minneapolis, MN, USA), N2 supplement (Gibco, Franklin Lakes, NJ, USA), and EGF (10 $\mathrm{ng} \mathrm{ml}^{-1}$; R\&D Systems). Cisplatin was purchased from Sigma-Aldrich (St Louis, MO, USA). The following primary antibodies were purchased from Santa Cruz Biotechnology (Santa Cruz, CA, USA): anti-Id2, anti-Oct4, antiSox2, anti-CD44, anti-cyclin A, anti-cyclin B, anti-cyclin D1, and anti-ATP-binding cassetteG2 (ABCG2). The following secondary antibodies were purchased from Jackson ImmunoResearch Laboratories (West Grove, PA, USA): anti-rabbit IgG and antimouse IgG.

Plasmid transfection. Short hairpin RNA (shRNA) targeting human Id2 was purchased from Sigma-. The following constructs were kindly provided by Prof. Kim H (Korea University, Seoul, South Korea): pBabe-Id2, Id2 overexpression, and pBabe-scramble. The shRNA-Id2 vector was transfected into KU-SCC-3 cells using a microporator apparatus. Transfection of the Id 2 overexpression vector was performed using the PolyExpress Transfection Reagent (Excellgen, Rockville, MD, USA) according to the manufacturer's instructions. Cells were selected for 2 weeks using the antibiotic puromycin (Calbiochem, Darmstadt, Germany) for the shId2 vector and G418 (Duchefa Biochemie, BH Haarlem, Netherlands) for the Id2 overexpression vector. For knockdown of cyclin A, small interfering RNAs were purchased from Bioneer (Daejeon, South Korea) and transfected using Lipofectamine (Life Technologies, Carlsbad, CA, USA).

Quantitative reverse transcription-polymerase chain reaction. Total RNA $(0.5 \mu \mathrm{g})$ was used to synthesise cDNA with a ReverTra Ace qPCR RT Master Mix and a gDNA Remover Kit (Toyobo Co. Ltd, Osaka, Japan) according to the manufacturer's instructions. For semi-quantitative reverse transcription-polymerase chain reaction (RT-PCR), the synthesised cDNA was added to a mixture of $1 \mathrm{U}$ of Taq DNA polymerase (Lucigen, Middleton, WI, USA) and gene-specific primers. This was amplified using the T100 Thermal Cycler (Bio-Rad Laboratories, Hercules, IN, USA). PCR products were separated by electrophoresis on $1.5 \%$ agarose gels and detected under ultraviolet light (Bio-Rad, Hercules, CA, USA). Real-time RT-PCR analysis was performed on a LightCycler 480 Real-Time Detection System (Roche Diagnostics, Indianapolis, IN, USA) using $2 \times$ LightCycler 480 SYBR-Green Master Mix (Roche Diagnostics). The sequences of human-specific primers used are:

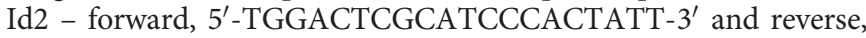
$5^{\prime}$-ATTCAGAAGCCTGCAAGGAC-3'; NGFR (nerve growth factor receptor) - forward, 5' - CTGCTGTTGCTGCTTCTGGG-3'; reverse, 5' - GGCTCACACACGGTCTGGTT-3'; CK-4 - forward, $5^{\prime}$-AGGAGGTCACCATCAACCAG-3' and reverse, $5^{\prime}$-ACCTTG TCGATGAAGGAGGC-3'.

Western blot analysis. Cells were lysed in a lysis buffer $(50 \mathrm{~mm}$ Tris- $\mathrm{HCl}$ of $\mathrm{pH}$ 7.5, 2 mм EDTA, $150 \mathrm{~mm} \mathrm{NaCl}, 1 \%$ Triton X-100, $1 \%$ sodium deoxycholate, $0.1 \%$ SDS, and a mixture of protease and phosphatase inhibitors) on ice for $30 \mathrm{~min}$. The lysates were centrifuged at 14000 r.p.m. at $4{ }^{\circ} \mathrm{C}$ for $20 \mathrm{~min}$, and the protein concentrations were determined using the Bradford protein assay 
(Bio-Rad Laboratories). Sodium dodecyl sulfate-polyacrylamide gel electrophoresis was used to separate the proteins, which were subsequently transferred to a polyvinylidene fluoride membrane (Millipore, Billerica, MA, USA). The membrane was blocked with $5 \%$ non-fat dry milk in Tris-buffered saline containing $0.1 \%$ Tween-20 (TBST) at room temperature for $1 \mathrm{~h}$, and incubated with primary antibodies overnight at $4{ }^{\circ} \mathrm{C}$. The membrane was washed the next day with TBST and incubated with the corresponding horseradish peroxidase-conjugated secondary antibody for $1 \mathrm{~h}$. Finally, immunoreactive bands were visualised by enhanced chemiluminescence detection.

Immunocytochemistry. Spheres were fixed in $4 \%$ paraformaldehyde, embedded and frozen in optimal cutting temperature compound, and cryosectioned (5 $\mu \mathrm{m}$ thick). Sections were immunostained with antibodies against Id2 (1:50; Santa Cruz Biotechnology) overnight at $4{ }^{\circ} \mathrm{C}$. After washing, sections were incubated with Cy3-conjugated anti-mouse secondary antibodies. Nuclei were counterstained with 4',6-diamidino-2-phenylindole (DAPI; $1 \mu \mathrm{g} \mathrm{ml}^{-1}$ ). Slides were photographed using an optical or confocal microscope (Zeiss, Oberkochen, Germany).

Cell growth and proliferation assay. To evaluate cell growth, $2 \times 10^{4}$ cells were plated and grown in media supplemented with FBS. Every 2 days, cells were detached with trypsin and the total number of cells was counted. Cell proliferation was measured using the colorimetric 3-(4,5-dimethylthiazol-2-yl)-5-(3-carboxymethoxyphenyl)-2-(4-sulphophenyl)- $2 \mathrm{H}$-tetrazolium (MTS) assay (Promega, Madison, WI, USA). Briefly, $5 \times 10^{4}$ cells were seeded into a 96-well plate. After incubation for $24 \mathrm{~h}, 20 \mu \mathrm{l}$ of MTS solution was added to each sample well in $100 \mu \mathrm{l}$ of culture medium and incubated at $37^{\circ} \mathrm{C}$ for $2 \mathrm{~h}$. Colorimetric analysis was performed using an ELISA Plate Reader (Molecular Devices, Sunnyvale, CA, USA) at $490 \mathrm{~nm}$.

Flow cytometry for detection of CD44 levels. Spheroid cells were dissociated into single cells, washed, and suspended in PBS. Cells were incubated with an anti-CD44 primary antibody and a FITClabelled secondary antibody, and subjected to flow cytometry using a FACSCalibur machine (BD Biosciences, San Jose, CA, USA).

Sphere-forming assay. To assess self-renewal in vitro, cells were dissociated into single cells, seeded into a 24 -well plate at a density of 500 cells per well, and cultured in serum-free media with EGF and bFGF supplementation every other day. Spheres with a diameter exceeding $50 \mu \mathrm{m}$ were counted after 7 days.

Cell viability assay for cisplatin. Cells were plated in a 96-well plate at a density of $1 \times 10^{4}$ cells per well, and then treated with either cisplatin at various concentrations or DMSO. Two days later, $10 \mu \mathrm{l}$ of 3-(4,4-dimethylthiazol-2-yl)-2,5-diphenyltetrazolium bromide (MTT) solution ( $5 \mathrm{mg} \mathrm{ml}^{-1}$ in PBS) was added to each well and incubated at $37^{\circ} \mathrm{C}$ for $2 \mathrm{~h}$. The absorbance was measured using a SpectraMax 190 (Molecular Devices) at a wavelength of $570 \mathrm{~nm}$.

In vivo xenograft experiments. All animal studies were approved by the Institutional Animal Care and Use Committee of Konkuk University. To assess the tumourigenicity of HNSCC cells treated with Id 2 shRNA or control shRNA, dissociated spheroid cells were counted, resuspended in $100 \mu \mathrm{l}$ of Matrigel, and injected subcutaneously into the flank of 8 -week-old female BALB/c nude mice using a 22-gauge needle. Engrafted mice were inspected weekly for signs of tumour appearance by visual observation and palpation for 8 weeks following the injection.

Patient sample immunohistochemistry. Immunohistochemistry of HNSCC patients' tissues was approved by the local institutional review board. HNSCC samples were collected from patients who had given informed consent. Formalin-fixed and paraffinembedded tissues from 119 HNSCC lesions were retrieved from the archives of the Pathology Department of Chungnam National University Hospital in Korea. These included 44 samples from the oral cavity, 17 from the oropharynx, 48 from the larynx, and 10 from the hypopharynx. Paraffin-embedded tissues were section at $4 \mu \mathrm{m}$ and used for immunohistochemical staining according to the methods provided in the Vectastain ABC Kit (Vector, Burlingame, CA, USA). The intensity of immunostaining was then analysed. Briefly, the intensity was scored as grade 0 when no positive cells were identified, grade 1 with weak staining, grade 2 with moderate staining, and grade 3 with strong staining. For statistical analyses, negative staining was considered to be grade 0 or grade 1 staining, and positive staining was considered to be grade 2 or grade 3 staining. All slides were scored by at least two pathologists who had no clinical information about the patients.

Statistics. All analyses were performed using SPSS 12.0 (SPSS Inc., Chicago, IL, USA). Statistical significance was assessed using a twotailed Student's $t$-test or ANOVA test. The association between Id2 expression and clinical parameters was analysed by Fisher's exact test. The survival analysis in human and mice samples was performed using the Kaplan-Meier survival curve and log-rank test. $P^{\star}<0.05$ was considered statistically significant.

\section{RESULTS}

Id genes are differentially expressed in HNSCC cells. First, we identified which subtype of the four Id gene families (Id1-Id4) is enriched in HNSCC cells. We performed qRT-PCR to examine the transcript levels of various Id genes in spheroid form and the attached form of HNSCC cells, because any cells that produce the spheroid form under serum-free conditioned media have the characteristics of CSCs (Jeon et al, 2008; Lim et al, 2011). Interestingly, Id 2 mRNA levels were markedly elevated in spheroid form of HNSCC cells compared with the expression of the other Id genes (Supplementary Figure S1). Thus, we hypothesise that Id2 expression may correlate with phenotypic characteristics associated with HNSCC CSCs, and performed the further experiments.

Overexpression of Id 2 accelerates HNSCC cell growth via cyclin A activation. To evaluate the effect of Id 2 overexpression on the proliferation of HNSCC cells, we generated Id2-overexpressing $\mathrm{FaDu}$ and SNU1041 cells (Supplementary Figure S2A). Id2 overexpression significantly increased the growth of $\mathrm{FaDu}$ (Figure 1A and Supplementary Figure S2B) and SNU 1041 cells (Figure $1 \mathrm{~B}$ and Supplementary Figure S2C) in vitro. It also significantly increased the sizes and weights of tumours generated after inoculation of mice with transfected FaDu cells (Figure 1C). Next, we determined whether any cyclins correlated with the increased cell proliferation of the Id2-overexpressing $\mathrm{FaDu}$ and SNU1041 cells. We found that an elevation in cyclin A expression was observed in Id2-overexpressing cells (Figure 1D and Supplementary Figure S2D). Furthermore, siRNA-mediated depletion of cyclin A in Id2-overexpressing FaDu cells (Supplementary Figure S2E) decreased the proliferation of tumours produced by Id2-overexpressing FaDu cells (Supplementary Figure S2F and G). Therefore, the proliferation activity of Id 2 seems to be mediated, in part, through cyclin A-driven cell proliferation. Taken together, ectopic Id2 expression promotes the tumour growth of HNSCC cells through the activation of cyclin A.

Overexpression of Id2 enhances stemness feature of HNSCC cells. One of the key traits of a CSC is its self-renewal capacity (AlHajj et al, 2003). To examine this in HNSCC, we compared the self-renewal capacity of Id2-overexpressing cells to control cells using a sphere-forming assay. The number of spheres was significantly higher in Id2-overexpressing HNSCC cells compared with control cells (Figure $2 \mathrm{~A}$ and $\mathrm{B}$ ). We then compared the 
A

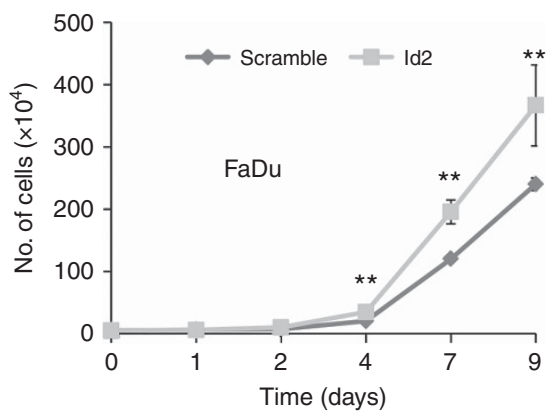

C

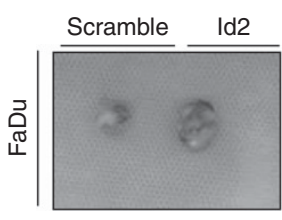

B

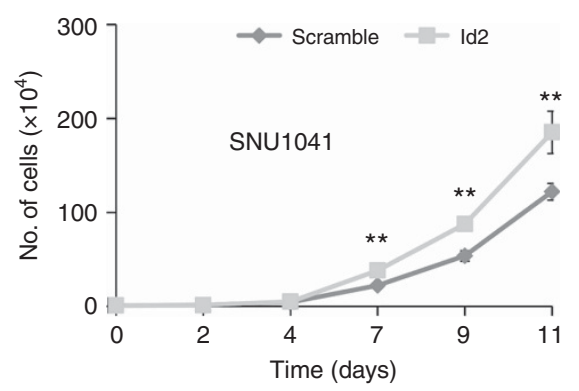

D

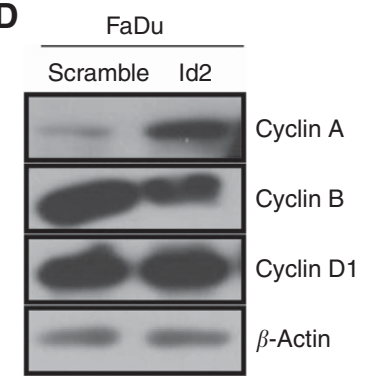

Figure 1. Inhibitor of DNA binding 2 overexpression enhances tumour growth of HNSCC cells by increasing cyclin A expression. (A) Cell proliferation rates in FaDu cells transfected with either ld2 or control vector. Data represent the means \pm s.d. ( $N=3$ ). (B) Cell proliferation rates in SNU1041 cells transfected with either Id2 or control vector. Data represent the means \pm s.d. $(N=3)$. (C) In vivo tumour growth capacity in FaDu cells transfected with either Id2 or control vector. Data represent the means \pm s.d. $(N=6)$. (D) Protein levels of various cyclin proteins in FaDu cells transfected with either ld2 or control vector. ${ }^{\star \star} P<0.01$.

expression levels of stem cell markers, including OCT4 and SOX2, in Id2-overexpressing cells versus control cells. We found that the protein levels of SOX2 were significantly higher in Id2-overexpressing HNSCC cells compared with control cells (Supplementary Figure S3A). Recently, Kang et al (2015) reported that NGFR and CK4 were suggested as stemness and differentiation markers in tonsillar epithelial stem cell, respectively. Thus, we examined mRNA levels of these genes in scrambled- and Id2overexpressing HNSCC cells. As a result, transcriptional levels of NGFR increased in Id2-overexpressing HNSCC cells, and that of CK5 decreased in same cells, compared with that in scrambled HNSCC cells (Supplementary Figure S3B). Previously, putative HNSCC CSCs have been isolated based on CD44 expression, which has been proposed to be a molecular marker for these cells (Prince et al, 2007). Thus, we investigated whether there were differences in CD44 expression between Id2-overexpressing cells and control cells. Id2-overexpressing HNSCC cells displayed higher levels of CD44 expression compared with control cells in vitro and in vivo (Figure $2 \mathrm{C}$ and $\mathrm{D}$ ). Taken together, our data suggest that Id 2 expression may enhance stemness feature in HNSCC cells.

Knockdown of Id 2 gene expression reduces stemness of primary HNSCC cells. Inhibitor of DNA binding 2 expression levels are also increased in the spheroid form of primary HNSCC cells generated from the HNSCC tumour tissue (Figure 3A and B and Supplementary Figure S4A). To elucidate the role of Id 2 in HNSCC CSC maintenance, we examined the effects of Id 2 knockdown on the stemness of primary HNSCC cells (Supplementary Figure S4B). Targeting Id2 with shRNA reduced the efficiency of sphere formation and decreased the expression of SOX2 and CD44 in primary HNSCC cells (Figure 3C and D and Supplementary Figure S5). These data demonstrate that depletion of Id2 specifically disrupts stemness traits of primary HNSCC cells.

Id2 expression is associated with cisplatin resistance in HNSCC cells. Cancer stem cells are believed to be highly resistant to conventional chemotherapeutic drugs (Dean et al, 2005).
Therefore, we examined whether Id 2 has a role in chemoresistance in HNSCC cells. Cell viability of Id2-overexpressing HNSCC cells after cisplatin treatment was significantly higher than in scrambled control cells (Supplementary Figure S6A). In addition, a reduction in Id 2 expression in primay HNSCC cells markedly decreased chemoresistance to cisplatin compared with the scrambled control cells (Supplementary Figure S6B). One of the mechanisms mediating CSC chemoresistance is overexpression of the ATPbinding cassette $(\mathrm{ABC})$ transporters, which regulate the flux of chemotherapeutic agents across the plasma membrane (Cui et al, 2015). Of these ABC transporters, ABCG2 overexpression is associated with multidrug resistance and cancer progression in HNSCC (Zhao et al, 2015). Thus, we examined whether Id2 chemoresistance correlates with ABCG2 expression in HNSCC cells. Interestingly, we found that Id2 knockdown reduced ABCG2 expression in HNSCC cells (Supplementary Figure S6C). These data suggest that $\mathrm{Id} 2$ expression is associated with cisplatin resistance in HNSCC cells.

Knockdown of Id2 suppresses HNSCC tumour growth in a xenograft mouse model. To investigate the effect of Id 2 on the tumourigenicity of HNSCC cells in vivo, we used a xenograft model to assess tumour formation after knockdown of Id2. We subcutaneously injected different amounts of HNSCC-shId 2 or HNSCC-scramble shRNA cells $\left(5 \times 10^{3}\right.$ or $5 \times 10^{4}$ cells $)$ into nude mice. Surprisingly, even $5 \times 10^{3}$ HNSCC-scramble shRNA cells were sufficient to initiate tumour formation. However, none of the mice injected with $5 \times 10^{3}$ HNSCC-shId 2 cells exhibited visible tumour formation (Figure $4 \mathrm{~A}$ ). In addition, the average weight of tumours generated in mice after inoculation with $5 \times 10^{4}$ HNSCC-shId2 cells was significantly lower compared with the control groups (Figure 4B). Furthermore, mice inoculated with HNSCC-shId2 cells had significantly increased survival rates compared with the control groups (Figure 4C). Taken together, our findings suggest that Id2 has an important role in HNSCC cells tumour initiation in a xenograft mouse model. 
A

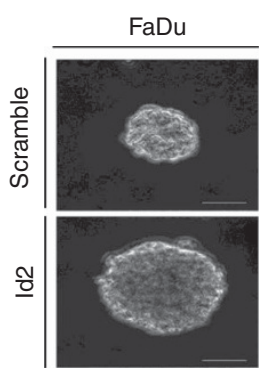

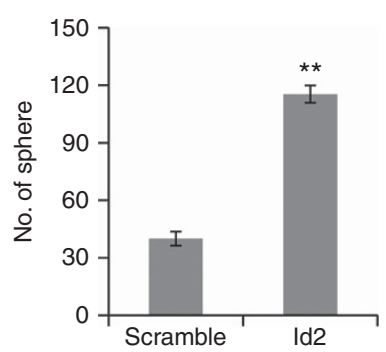

B
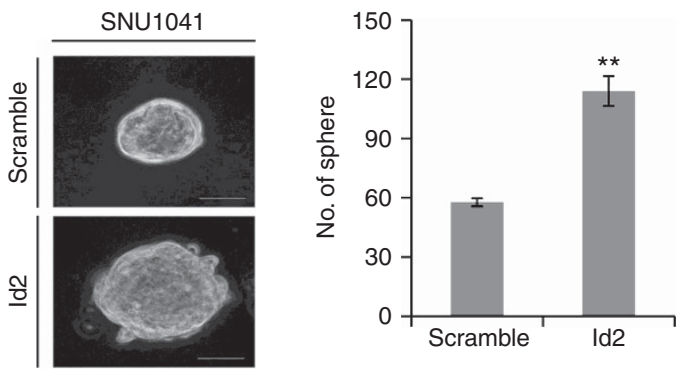

C
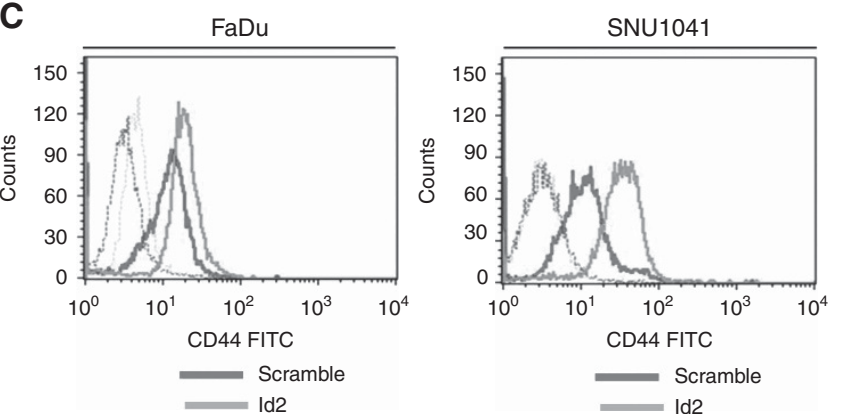

D

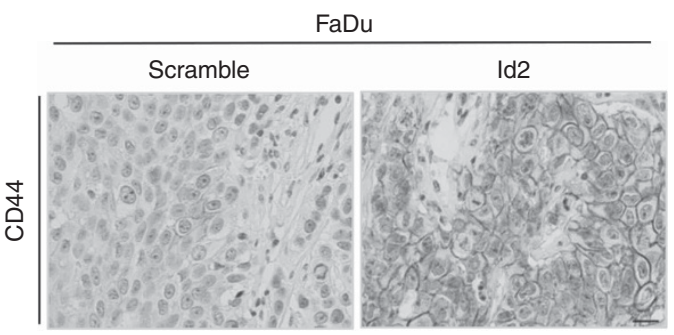

Figure 2. Inhibitor of DNA binding overexpression enhances HNSCC stemness. (A) Sphere formation capacity of FaDu cells transfected with either Id2 or control vector. Scale bar, $30 \mu \mathrm{m}$. Data represent the means \pm s.d. $(N=3)$. (B) Sphere formation capacity of SNU1041 cells transfected with either Id2 or control vector. Scale bar, $30 \mu \mathrm{m}$. Data represent the means \pm s.d. $(N=3)$. (C) In vitro expression levels of CD44 in HNSCC cells transfected with either Id2 or control vector. (D) In vivo expression levels of CD44 in tumour tissue generated by inoculation of Id2-FaDu cells in nude mice. ${ }^{\star \star} \mathrm{P}<0.01$.

A

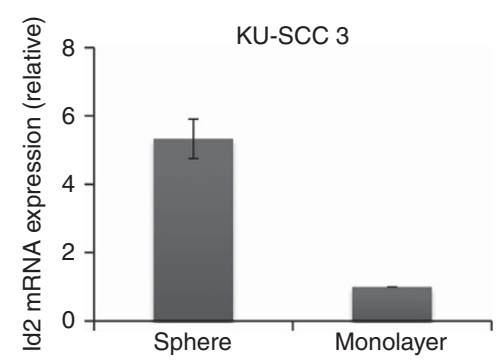

C

KU-SCC 3

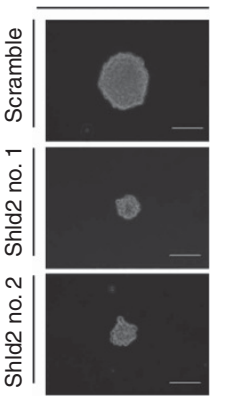

B
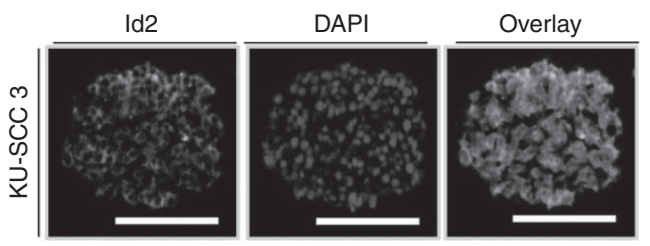

D

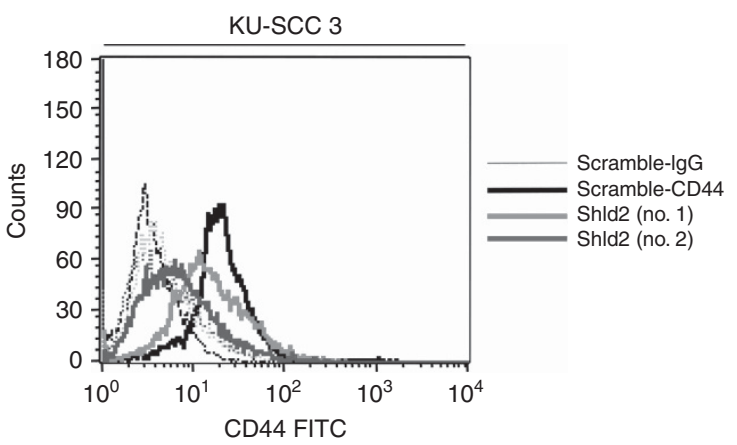

Figure 3. Knockdown of Id2 decreases stemness of primary HNSCC cells. (A) Messenger RNA levels of Id2 during sphere formation and in attached (monolayer) primary HNSCC cells. (B) Immunofluorescent staining of Id2 in sphere-forming primary HNSCC cells. Scale bar, $100 \mu \mathrm{m}$. (C) Sphere formation capacity of primary HNSCC cells transfected with either shld2 or control vector. Scale bar, $30 \mu \mathrm{m}$. Data represent the means \pm s.d. $(N=3)$. (D) Expression levels of CD44 in primary HNSCC cells transfected with either shld2 or control vector. ${ }^{\star \star} P<0.01$.

Id 2 expression is a valuable prognostic indicator in patients with HNSCC. To address whether Id2 expression is clinically relevant in HNSCC patients, 119 samples from patients with primary HNSCCs were evaluated using antibodies against Id2 (Supplementary Table S1). Immunohistochemical study revealed that specific Id 2 protein staining was found in the cytoplasm and nucleus of tumour cells (Supplementary Figure S7) HNSCC patients displaying high levels of Id 2 expression had significantly lower survival rates compared with those with lower expression levels $(P<0.001)$ (Figure 5A and $\mathrm{B})$. The median 5-year overall and cancer-specific survival rates for patients possessing tumours with high and low Id 2 expression were $39.9 \%$ and $84.2 \%$ and $42.6 \%$ 
A

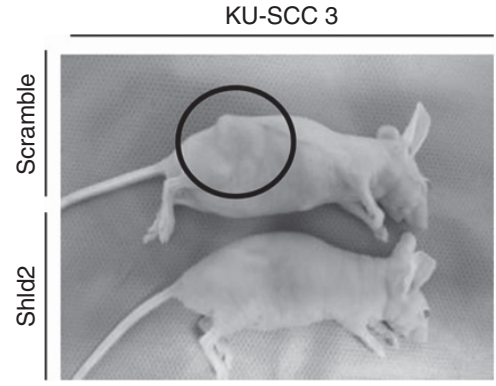

B

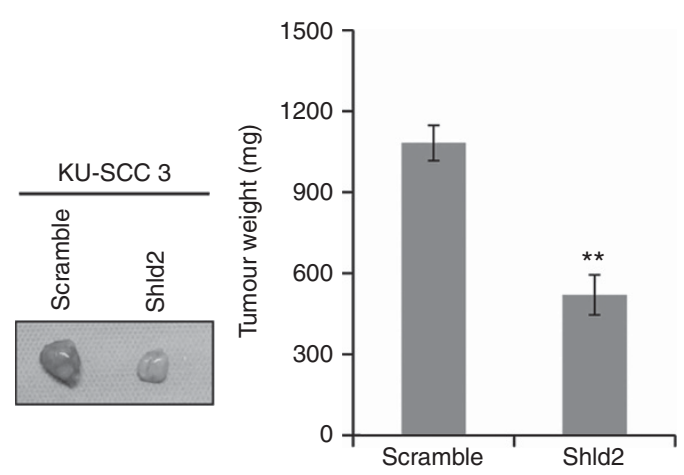

\begin{tabular}{|c|c|}
\hline No. of injected cells & Tumour formation \\
\hline \multicolumn{2}{|l|}{ Scramble } \\
\hline $5 \times 10^{3}$ & $5 / 6$ \\
\hline $5 \times 10^{4}$ & $6 / 6$ \\
\hline \multicolumn{2}{|l|}{ Shld2 } \\
\hline $5 \times 10^{3}$ & $0 / 6$ \\
\hline $5 \times 10^{4}$ & $2 / 6$ \\
\hline
\end{tabular}

C

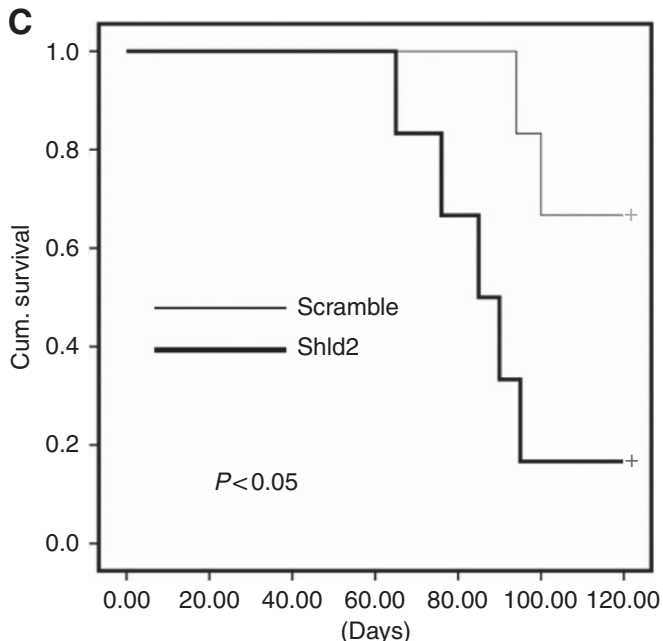

Figure 4. Knockdown of Id2 suppresses HNSCC tumour growth and increases survival rates of mice receiving HNSCC cell xenografts. (A) In vivo propagation of human head and neck cancers in nude mice by xenografts of HNSCC cells transfected with either shScramble or shld2 construct. Representative photos showing a tumour in a mouse at the cell injection site. (B) Average weights of tumours generated after subcutaneous injection of $5 \times 10^{4} \mathrm{HNSCC}$ cells transfected with either shScramble $(N=6)$ or shld2 construct $(N=2)$. (C) Kaplan-Meier survival curves for mice implanted with HNSCC cells transfected with either shScramble or shld2 construct $(N=6) .{ }^{\star \star} P<0.01$.

A

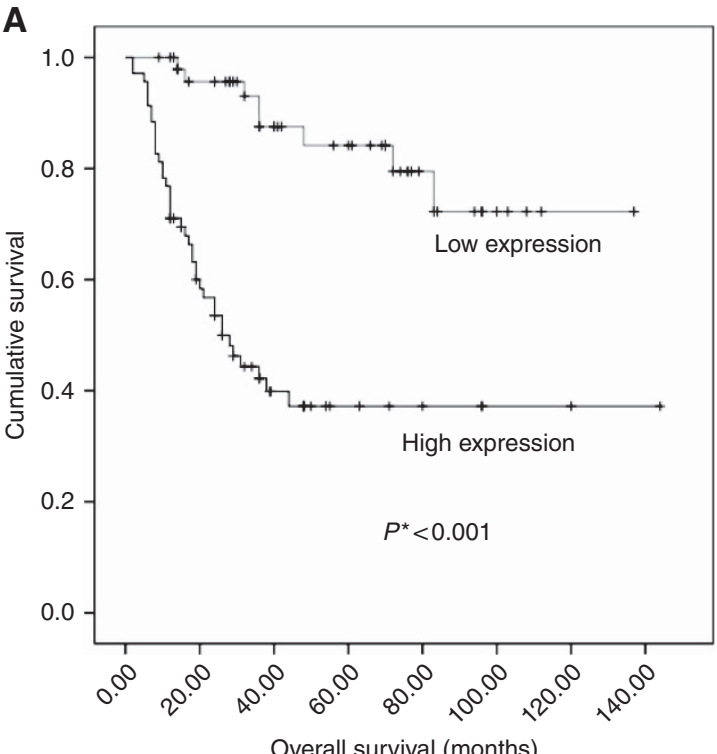

B

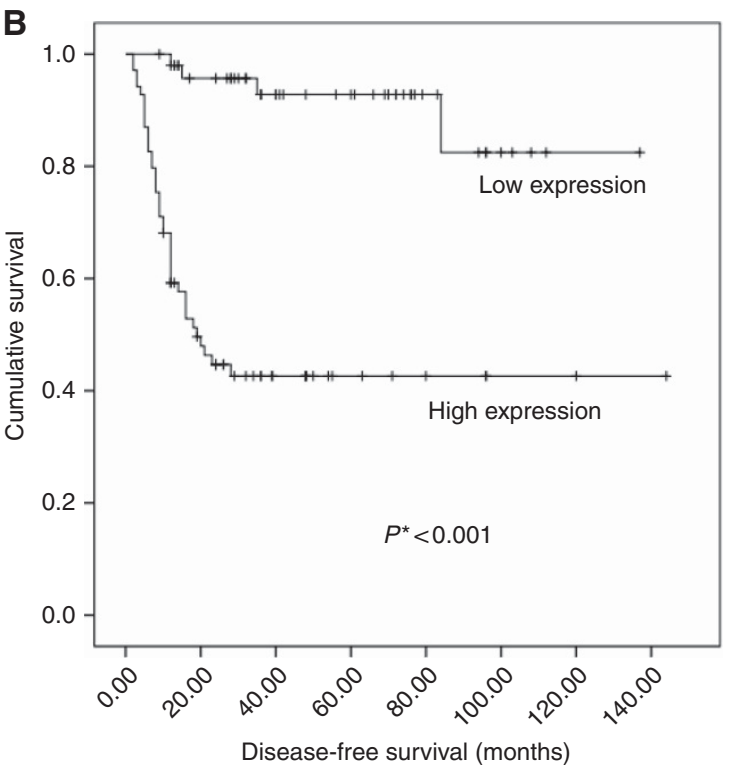

Figure 5. Inhibitor of DNA binding 2 expression levels correlate with survival rates of human HNSCC patients. (A) Overall survival rates based on Id2 expression $(N=119)$. (B) Disease-specific survival rates based on Id2 expression $(N=119)$.

and $92.8 \%$, respectively $(P<0.001)$. The association of various clinicopathological factors influencing the prognosis of HNSCC patients with Id 2 expression was examined (Supplementary Table S2 and Tables 1 and 2). Univariate analysis showed a statistically significant increase in Id2 expression in advanced tumour stages (T), advanced AJCC (American Joint Committee on Cancer) stages, poor histologic grades, and in the presence of lymph node metastasis (Supplementary Table S2). Multivariate analysis showed 
Table 1. Multivariate analysis of the relationship between Id2 expression and clinicopathologic factors

\begin{tabular}{|c|c|c|c|c|}
\hline Factors & $\beta$ (s.e.) & $P$-value & $\operatorname{Exp}(\beta)$ & $95.0 \% \mathrm{Cl}$ \\
\hline Advanced T stage & $0.119(0.734)$ & 0.872 & 1.126 & $(0.267,4.750)$ \\
\hline Lymph node metastasis & $2.539(0.666)$ & $0.000^{\mathrm{a}}$ & 12.673 & $(3.435,46.757)$ \\
\hline Advanced AJCC stage & $0.232(0.910)$ & 0.799 & 1.261 & $(0.212,7.508)$ \\
\hline Poor histologic grade & $1.039(0.571)$ & 0.069 & 2.827 & $(0.924,8.650)$ \\
\hline \multicolumn{5}{|c|}{$\begin{array}{l}\text { Abbreviations: } \mathrm{AJCC}=\text { American Joint Committee on Cancer; } \mathrm{Cl}=\text { confidence interval; } \\
\operatorname{Exp}(\beta)=\text { Odds ratio; } \mathrm{Id} 2=\text { inhibitor of DNA binding } 2 . \\
{ }^{\mathrm{a}} \mathrm{P}<0.05 \text {. }\end{array}$} \\
\hline
\end{tabular}

Table 2. Multivariate Cox's regression analysis of diseasespecific death events related to 119 HNSCC patients

\begin{tabular}{|l|c|c|c|}
\hline Parameters & RR & $\mathbf{9 5 \% ~ C l}$ & $\boldsymbol{P}$-value \\
\hline Age & 0.924 & $0.481-1.774$ & 0.812 \\
\hline Sex & 0.453 & $0.130-1.586$ & 0.216 \\
\hline Advanced T stage & 0.649 & $0.320-1.314$ & 0.229 \\
\hline Lymph node metastasis & 1.276 & $0.546-2.980$ & 0.573 \\
\hline Advanced AJCC stage & 4.639 & $1.119-19.240$ & $0.034^{\mathrm{a}}$ \\
\hline Poor histologic grade & 6.193 & $1.433-26.760$ & $0.015^{\mathrm{a}}$ \\
\hline Intense Id2 expression & 5.085 & $1.647-15.701$ & $0.005^{\mathrm{a}}$ \\
\hline
\end{tabular}

Abbreviations: $\mathrm{AJCC}=$ American Joint Committee on Cancer; $\mathrm{Cl}=$ confidence interval; $\mathrm{Id} 2=$ inhibitor of DNA binding 2; $\mathrm{RR}=$ ratio of risk.

${ }^{\mathrm{a}} \mathrm{P}<0.05$.

that the presence of lymph node metastases was independent of high Id2 expression $(P<0.01)$ (Table 1). Today, the most commonly used indicator to predict the prognosis of patients with HNSCC after primary treatment in a clinical setting is the AJCC staging system. Therefore, we evaluated whether Id2 expression predicts the survival rates of HNSCC patients after treatment more reliably than the AJCC system. Notably, we found that the intensity of Id 2 expression was better than the AJCC system at predicting the likelihood of survival in HNSCC patients after primary treatment (Table 2). Taken together, our data suggest that Id2 expression is a valuable prognostic indicator for these patients.

\section{DISCUSSION}

Since the discovery of HNSCC CSCs by Prince et al (2007), some proteins, including those on the cell surface, are being investigated as potential molecular markers of HNSCC CSCs. However, there is a paucity of data regarding the intracellular signalling molecules specific to CSCs and the signalling pathways controlling their stemness. Cancer stem cells share characteristics with normal tissue stem cells, such as self-renewal and multipotency. Thus, the dysregulation of pathways required to maintain stemness in normal stem cells may contribute to the development of CSCs, and key factors contributing to this dysregulation may provide a critical clue to the mechanisms of CSC genesis.

In embryonic and somatic stem cells, in addition to their progenitors, Id family members are required to preserve stem cell identity and prevent premature differentiation (Massari and Murre, 2000). In embryonic stem cells, pluripotency is sustained by bone morphogenetic protein 4-mediated expression of Id genes via SMAD signalling (Ying et al, 2003). Additionally, in neural stem cells and their progenitors, multiple Id family members maintain stem cell identities (Sullivan et al, 2016). Also, the epithelium of the distal tip of the lung expresses Id 2 and contains multipotent embryonic progenitors (Rawlins et al, 2009).
As mentioned in the Introduction, Id genes are frequently dysregulated in many types of human neoplasms. They function as 'hubs' for the crosstalk of multiple cancer signalling pathways. In head and neck cancers, Id protein immunoreactivity was observed in the majority of malignant keratinocytes in the most poorly differentiated sections, while reduced staining was found in welldifferentiated tumours (Langlands et al, 2000). Id1 protein expression also correlates with lymph node status, indicating that increased Id expression may be related to the metastatic behaviour of human oral SCC (Dong et al, 2010). Patients with increased Id2 expression levels had poorer overall survival rates than those with low Id2 levels in nasopharyngeal carcinoma (Liu et al, 2012). This suggests that overexpression of Id2 protein is an unfavourable prognostic factor that indicates likely cell proliferation in nasopharyngeal carcinoma.

These aforementioned studies indicated that Id proteins are likely to be an important and intrinsic determinant of CSCs, possibly conferring traits associated with both cancer cells and normal stem cells.

To date, possible functions for Id proteins in CSCs have been explored in various solid tumours, including colon cancer, glioma, breast cancer, and prostate cancer. In colon CSCs, the combined expression of Id 1 and Id 3 increased both self-renewal and tumour initiation (O'Brien et al, 2012). Meanwhile, Id4 induced expression of glioma stem cell markers, likely through the activation of cyclin E and Notch signalling (Jeon et al, 2008). Furthermore, Id1 was found to be a key transcriptional regulator for multiple tumourpromoting pathways in glioblastoma (Anido et al, 2010). The four Id genes are located on different chromosomes and have different expression patterns and functions. Studies of Id 2 and its potential roles in CSC genesis suggested that a link between Id2 expression and CSC phenotypes is unlikely. However, two conflicting articles regarding the function of $\mathrm{Id} 2$ with respect to CSCs was recently published. Dong et al (2016) suggested that $\mathrm{Wnt} / \beta$-catenin signalling mediated hypoxia-induced self-renewal potential of colorectal CSCs by reactivating Id 2 expression. In contrast, Ghisi et al (2016) showed that low endogenous Id2 expression is associated with leukaemia stem cell enrichment, while Id2 overexpression inhibits the initiation and growth of mixed lineage leukaemia-AF9 leukaemia. Thus, it is still poorly understood how Id2 influences CSCs. In this study, we show for the first time that Id 2 expression may promote the establishment and maintenance of stemness in CSCs generated from HNSCC cell lines and primary cultures of HNSCC tissues. Our findings establish that overexpression of Id 2 increases self-renewal and chemoresistance of differentiated HNSCC cells. Moreover, silencing this gene in primary HNSCC cells results in a critical loss of tumour-initiating potential in xenograft mouse models.

It has been suggested that Id proteins control normal and abnormal cell cycle progression, by binding to important cell cycle regulatory proteins (Zebedee and Hara, 2001). Interestingly, our study revealed that Id 2 increased cyclin A expression. Liu et al (2000) showed that wild-type Id2 controlled the activity of the cyclin A promoter in A549 lung epithelial cells, implying that Id2 could affect cell-cycle gene transcription. Mechanistic insights into the role of Id 2 regulating cell cycle of HNSCC cells will be needed for further studies. Several Id-dependent targets are implicated in stem cell renewal, such as NANOG, HES1, NOTCH, SOX2, and LIF (Lasorella et al, 2014). Of these, Galatro et al (2013) showed that Id4 expression is associated with the induction of SOX. Jeon et al (2011) also demonstrated that Id4 inhibits miR- $9^{\star}$-mediated suppression of SOX2, which leads to increased glioma stem cell potential and chemoresistance. In addition, our previous study showed that SOX2 has a crucial role in the genesis and maintenance of HNSCC CSCs (Lee et al, 2014). In the present study, overexpression of Id 2 increased SOX2 expression. Thus, Id 2 may promote the stemness of HNSCC cells through SOX2 
activation. Further verification about these relationship will be needed.

One particularly notable finding from our study is the importance of $\mathrm{Id} 2$ expression as a prognostic factor. In a clinical setting, physicians must predict the prognosis of HNSCC patients before and after treatment using the TNM staging system, which assesses the primary tumour size $(\mathrm{T})$, the presence of lymph node metastasis $(\mathrm{N})$, and the presence of distant metastasis (M). To our knowledge, this system is the most commonly used assay for predicting clinical outcomes for HNSCC patients. However, patients with early-stage cancer (stage I) may die because of cancer recurrence, while patients with advancedstage cancer (stage IV) may be cured without cancer recurrence. Thus, the TNM staging system has clear limitations. The present study compared the accuracy of TNM staging with the use of Id 2 expression strength for predicting the prognosis of HNSCC patients. Our results suggest that Id expression is a better indicator of patient survival compared with the TNM staging system.

Recent developments in identifying CSCs in solid tumours may provide tailored approaches to targeting CSCs. Recently, various intracellular factors and signalling pathways that maintain stemness in CSCs have been actively discussed. Therefore, as this study has identified Id 2 as a novel factor regulating HNSCC CSCs, future efforts should explore the development of a therapeutic agent targeting Id 2 as a potential treatment for HNSCC.

\section{ACKNOWLEDGEMENTS}

This study was supported by a National Research Foundation of Korea (NRF) grant funded by the Korean government (MEST) (No. 2015R1A2A2A01006688 and NRF-2016R1A5A2012284 to YCL, No. 2014R1A1A1004203 to WJB, and No. 2016R1A2A2A 05005220 to BSK). This study was also supported by the Korea Health Technology R\&D Project through the Korea Health Industry Development Institute (KHIDI), funded by the Ministry of Health \& Welfare, Republic of Korea (HI15C2807 to YCL).

\section{CONFLICT OF INTEREST}

The authors declare no conflict of interest.

\section{REFERENCES}

Al-Hajj M, Wicha MS, Benito-Hernandez A, Morrison SJ, Clarke MF (2003) Prospective identification of tumorigenic breast cancer cells. Proc Natl Acad Sci USA 100: 3983-3988.

Anido J, Sáez-Borderías A, Gonzàlez-Juncà A, Rodón L, Folch G, Carmona MA, Prieto-Sánchez RM, Barba I, Martínez-Sáez E, Prudkin L, Cuartas I, Raventós C, Martínez-Ricarte F, Poca MA, García-Dorado D, Lahn MM, Yingling JM, Rodón J, Sahuquillo J, Baselga J, Seoane J (2010) TGF- $\beta$ receptor inhibitors target the CD44(high)/Id1(high) gliomainitiating cell population in human glioblastoma. Cancer Cell 18: 655-668.

Bhaijee F, Pepper DJ, Pitman KT, Bell D (2012) Cancer stem cells in head and neck squamous cell carcinoma: a review of current knowledge and future applications. Head Neck 34: 894-899.

Cui H, Zhang AJ, Chen M, Liu JJ (2015) ABC transporter inhibitors in reversing multidrug resistance to chemotherapy. Curr Drug Targets 16: 1356-1371.

Dean M, Fojo T, Bates S (2005) Tumour stem cells and drug resistance. Nat Rev Cancer 5: 275-284.

Desprez PY, Sumida T, Coppé JP (2003) Helix-loop-helix proteins in mammary gland development and breast cancer. J Mammary Gland Biol Neoplasia 8: 225-239.
Dong HJ, Jang GB, Lee HY, Park SR, Kim JY, Nam JS, Hong IS (2016) The $\mathrm{Wnt} / \beta$-catenin signaling/Id2 cascade mediates the effects of hypoxia on the hierarchy of colorectal-cancer stem cells. Sci Rep 6: 22966.

Dong Z, Liu S, Zhou C, Sumida T, Hamakawa H, Chen Z, Liu P, Wei F (2010) Overexpression of Id-1 is associated with tumor angiogenesis and poor clinical outcome in oral squamous cell carcinoma. Oral Oncol 46: $154-157$.

Galatro TF, Uno M, Oba-Shinjo SM, Almeida AN, Teixeira MJ, Rosemberg S, Marie SK (2013) Differential expression of ID4 and its association with TP53 mutation, SOX2, SOX4 and OCT-4 expression levels. PLoS One 8: e61605.

Ghisi M, Kats L, Masson F, Li J, Kratina T, Vidacs E, Gilan O, Doyle MA, Newbold A, Bolden JE, Fairfax KA, de Graaf CA, Firth M, Zuber J, Dickins RA, Corcoran LM, Dawson MA, Belz GT, Johnstone RW (2016) Id2 and $\mathrm{E}$ proteins orchestrate the initiation and maintenance of MLLrearranged acute myeloid leukemia. Cancer Cell 30: 59-74.

Jeon HM, Jin X, Lee JS, Oh SY, Sohn YW, Park HJ, Joo KM, Park WY, Nam DH, DePinho RA, Chin L, Kim H (2008) Inhibitor of differentiation 4 drives brain tumor-initiating cell genesis through cyclin $\mathrm{E}$ and notch signaling. Genes Dev 22: 2028-2033.

Jeon HM, Sohn YW, Oh SY, Kim SH, Beck S, Kim S, Kim H (2011) ID4 imparts chemoresistance and cancer stemness to glioma cells by derepressing miR-9*-mediated suppression of SOX2. Cancer Res 71: 3410-3421.

Jung S, Park RH, Kim S, Jeon YJ, Ham DS, Jung MY, Kim SS, Lee YD, Park CH, Suh-Kim H (2010) Id proteins facilitate self-renewal and proliferation of neural stem cells. Stem Cells Dev 19: 831-841.

Kang SC, Kannan N, Zhang L, Martinez V, Rosin MP, Eaves CJ (2015) Characterization of epithelial progenitors in normal human palatine tonsils and their HPV16 E6/E7-induced perturbation. Stem Cell Rep 8: $1210-1225$.

Kong Y, Cui H, Zhang H (2011) Smurf2-mediated ubiquitination and degradation of Id1 regulates p16 expression during senescence. Aging cell 10: 1038-1046.

Langlands K, Down GA, Kealey T (2000) Id proteins are dynamically expressed in normal epidermis and dysregulated in squamous cell carcinoma. Cancer Res 60: 5929-5933.

Lasorella A, Benezra R, Iavarone A (2014) The ID proteins: master regulators of cancer stem cells and tumour aggressiveness. Nat Rev Cancer 14: 77-91.

Lee SH, Oh SY, Do SI, Lee HJ, Kang HJ, Rho YS, Bae WJ, Lim YC (2014) SOX2 regulates self-renewal and tumorigenicity of stem-like cells of head and neck squamous cell carcinoma. Br J Cancer 111: 2122-2130.

Leemans CR, Braakhuis BJ, Brakenhoff RH (2011) The molecular biology of head and neck cancer. Nat Rev Cancer 11: 9-22.

Lim YC, Oh SY, Cha YY, Kim SH, Jin X, Kim H (2011) Cancer stem cell traits in squamospheres derived from primary head and neck squamous cell carcinomas. Oral oncol 47: 83-91.

Liu J, Shi W, Warburton D (2000) A cysteine residue in the helix-loop-helix domain of Id2 is critical for homodimerization and function. Biochem Biophys Res Commun 273: 1042-1047.

Liu Z, Chen J, Luo W, Yang H, Wu A, Zhen Y, Yu X, Wang H, Yao K, Li X, Fang W (2012) Overexpressed DNA-binding protein inhibitor 2 as an unfavorable prognosis factor promotes cell proliferation in nasopharyngeal carcinoma. Acta Biochim Biophys Sin (Shanghai) 44: 503-512.

Love C, Sun Z, Jima D, Li G, Zhang J, Miles R, Richards KL, Dunphy CH, Choi WW, Srivastava G, Lugar PL, Rizzieri DA, Lagoo AS, Bernal-Mizrachi L, Mann KP, Flowers CR, Naresh KN, Evens AM, Chadburn A, Gordon LI, Czader MB, Gill JI, Hsi ED, Greenough A, Moffitt AB, McKinney M, Banerjee A, Grubor V, Levy S, Dunson DB, Dave SS (2012) The genetic landscape of mutations in Burkitt lymphoma. Nat Genet 44: 1321-1325.

Massari ME, Murre C (2000) Helix-loop-helix proteins: regulators of transcription in eucaryotic organisms. Mol Cell Biol 20: 429-440.

Matsui WH (2016) Cancer stem cell signaling pathways. Medicine (Baltimore) 95: S8-S19.

Niola F, Zhao X, Singh D, Castano A, Sullivan R, Lauria M, Nam HS, Zhuang Y, Benezra R, Di Bernardo D, Iavarone A, Lasorella A (2012) Id proteins synchronize stemness and anchorage to the niche of neural stem cells. Nat Cell Biol 14: 477-487. 
O’Brien CA, Kreso A, Ryan P, Hermans KG, Gibson L, Wang Y, Tsatsanis A, Gallinger S, Dick JE (2012) ID1 and ID3 regulate the self-renewal capacity of human colon cancer-initiating cells through p21. Cancer Cell 21: 777-792.

Perk J, Iavarone A, Benezra R (2005) Id family of helix-loop-helix proteins in cancer. Nat Rev Cancer 5: 603-614.

Polanska H, Raudenska M, Gumulec J, Sztalmachova M, Adam V, Kizek R, Masarik M (2014) Clinical significance of head and neck squamous cell cancer biomarkers. Oral Oncol 50: 168-177.

Prince ME, Sivanandan R, Kaczorowski A, Wolf GT, Kaplan MJ, Dalerba P, Weissman IL, Clarke MF, Ailles LE (2007) Identification of a subpopulation of cells with cancer stem cell properties in head and neck squamous cell carcinoma. Proc Natl Acad Sci USA 104: 973-978.

Rawlins EL, Clark CP, Xue Y, Hogan BL (2009) The Id2 + distal tip lung epithelium contains individual multipotent embryonic progenitor cells. Development 136: 3741-3745.

Richter J, Schlesner M, Hoffmann S, Kreuz M, Leich E, Burkhardt B (2012) Recurrent mutation of the ID3 gene in Burkitt lymphoma identified by integrated genome, exome and transcriptome sequencing. Nat Genet 44: 1316-1320.

Riechmann V, Sablitzky F (1995) Mutually exclusive expression of two dominant-negative helix-loop-helix (dnHLH) genes, Id4 and Id3, in the developing brain of the mouse suggests distinct regulatory roles of these dnHLH proteins during cellular proliferation and differentiation of the nervous system. Cell Growth Differ 6: 837-843.
Sikder HA, Devlin MK, Dunlap S, Ryu B, Alani RM (2003) Id proteins in cell growth and tumorigenesis. Cancer Cell 3: 525-530.

Sullivan JM, Havrda MC, Kettenbach AN, Paolella BR, Zhang Z, Gerber SA, Israel MA (2016) Phosphorylation Regulates Id2 degradation and mediates the proliferation of neural precursor cells. Stem Cells 34: 1321-1331.

Wang X, Di K, Zhang X, Han HY, Wong YC, Leung SC, Ling MT (2008) Id-1 promotes chromosomal instability through modification of APC/C activity during mitosis in response to microtubule disruption. Oncogene 27: 4456-4466.

Ying QL, Nichols J, Chambers I, Smith A (2003) BMP induction of Id proteins suppresses differentiation and sustains embryonic stem cell self-renewal in collaboration with STAT3. Cell 115: 281-292.

Zebedee Z, Hara E (2001) Id proteins in cell cycle control and cellular senescence. Oncogene 20: 8317-8325.

Zhao L, Ren Y, Tang H, Wang W, He Q, Sun J, Zhou X, Wang A (2015) Deregulation of the miR-222-ABCG2 regulatory module in tongue squamous cell carcinoma contributes to chemoresistance and enhanced migratory/invasive potential. Oncotarget 6: 44538-44550.

This work is published under the standard license to publish agreement. After 12 months the work will become freely available and the license terms will switch to a Creative Commons AttributionNonCommercial-Share Alike 4.0 Unported License.

Supplementary Information accompanies this paper on British Journal of Cancer website (http://www.nature.com/bjc) 